Kern ein um sich selbst kreisendes Sonderrecht". Es scheint an der Zeit, ,das Gesundheitswesen über punktuelle und kurzfristige Remeduren hinaus grundlegend neu auszurichten und dabei Individualität und Eigenverantwortung stärker zur Geltung zu bringen“ (Schmidt-ABmann, NJW 2004, 1690). In diesem Sinne unternimmt der Autor eine verfassungsrechtliche Fundamentalkritik, die sowohl aus der Perspektive der Ärzte wie aus der Sicht der sozialversicherten Patienten argumentiert.

Den Ausgangspunkt bietet das gesellschaftsgeschichtlich überlebte Sachleistungsprinzip, an dem das Leistungserbringungsrecht sich ausrichtet. Es verkürze den durch Art. 12 Abs. 1 GG dem Arzt eingeräumten Freiheitsraum zum einen durch das Zulassungserfordernis, eine Art zweiter Approbation, zum anderen durch Behandlungs- und Therapievorgaben. Die ,faktisch zwingende Eingliederung“ des Arztes in das System sei weder verhältnismäßig noch erforderlich, wie der Vergleich mit dem Kostenerstattungsprinzip der privaten Krankenversicherung zeige. Der vom BVerfG ,,als Rechtsgut von Verfassungsrang überhöhte Grundsatz der Stabilität der GKV" bilde hier keine verfassungsimmanente Grundrechtsschranke. Die Zwangsmitgliedschaft in Kassenärztlichen Vereinigungen wahre den Verhältnismäßigkeitsgrundsatz nicht (Art. 2 Abs. 1 GG). Die Zwangskorporationen seien nicht erforderlich und weder der Effektivität noch der Funktionsfähigkeit dienlich. Überdies verstießen ,zahlreiche sozialrechtliche Einflußnahmen auf das Arzt- bzw. Zahnarztrecht" gegen das legislative Kompetenzgefüge des GG. Ausführliche verfassungs- und europarechtliche Bedenken gegen die inzwischen wieder aufgehobenen Höchstaltersgrenzen schließen sich an.

Im Blick auf die sozialrechtliche Stellung der Versicherten erhebt der Autor wiederum verfassungsrechtliche Bedenken. Nach dem Prinzip der sozialstaatlichen Subsidiarität sei eine den weitaus größten Teil der Bevölkerung umfassende Pflichtmitgliedschaft in der GKV nicht verfassungskonform. Die sozialrechtliche Gestalt der Arztwahlfreiheit (Art. 2 Abs. 1 GG) stelle einen Eingriff dar, ebenso der prinzipielle Ausschluß der Kostenerstattungsmöglichkeiten bei der Wahl von Ärzten, die bei einem kollektiven Verlassen des Systems aus der vertragsärztlichen Versorgung ausschieden. Zweifelhaft sei die Verhältnismäßigkeit der normativ bindenden Wirkung der Erklärung über die Wahl der Kostenerstattung für ein Jahr.

Der Verfasser begründet die hier nur angedeuteten Einwände in nachvollziehbaren Gedankengängen. Das System der GKV erscheint jedenfalls in wesentlichen Zügen verfassungsrechtlich fragwürdig. Diese Bedenklichkeit mag die rechtspolitische Bereitschaft zu einer umfassenden Reform bestärken. Das Finanzierungsproblem bleibt aber außer Betracht, leider.

DOI: $10.1007 /$ s00350-010-2660-y

\section{Hypertrophie des ärztlichen Sozialrechts. Verfassungs- und europarechtliche Grenzen sozialrechtlicher Regelungen des (zahn-)ärztlichen Berufsrechts anhand ausgewählter Beispiele.}

Von Marc Schüffner und Laura Schnall. (Schriften zum Gesundheitsrecht, Bd. 18). Verlag Duncker u. Humblot, Berlin 2009, 94 S., kart., $€ 52,00$

Eine übermäßige Vergrößerung bei erhöhter Beanspruchung: so läßt sich das ärztliche Sozialrecht durchaus auf den Begriff bringen. Um die gebotenen Grenzen geht es in dem schmalen Buch auf dem Kurs, den der Herausgeber Helge Sodan, Direktor des Deutschen Instituts für Gesundheitsrecht und selbst Autor auf diesem Felde, vorgibt. Der Verfasser und die Verfasserin fahren ihrerseits schweres verfassungsrechtliches Geschütz auf, um wesentliche Elemente des Vertragsarztrechts zu Fall zu bringen. Die detaillierten Vorschriften des Vertragsarztrechtsänderungsgesetzes zur Berufsausübung überschritten, so meinen sie, die durch die klassische Sozialversicherung geprägte gesetzgeberische Zuständigkeit nach Art. 74 Nr. 12, Nr. 19, Nr. 19a GG. Die von $\$ 84$ Abs. 7a SGB V vorgesehene Wirtschaftlichkeitsprüfung mit der nicht widerspruchsfreien „Bonus-MalusRegel“" verstoße gegen das Rechtsstaatsprinzip sowie gegen Grundrechte von Ärzten und Patienten. Verfassungswidrig wegen ihrer

Prof. Dr. iur. Dr. h.c. Adolf Laufs, Heidelberg, Deutschland
Unverhältnismäßigkeit seien auch die gesetzlichen Sanktionen bei einem kollektiven Verzicht von Vertragsärzten auf ihre Zulassung nach $\$ 72 \mathrm{a}$ Abs. 1 SGB V. Schwerwiegende verfassungsrechtliche und systematische Probleme sehen der Autor und die Autorin im Sicherstellungsauftrag, den $\$ 75$ Abs. 3a SGB V für im Basistarif versicherte Personen statuiert. Schließlich erscheinen auch Höchstaltersgrenzen für vertragsärztliche Tätigkeiten grundrechtlich und außerdem europarechtlich fragwürdig.

Das Sozialrecht hat seine ursprüngliche Gestalt mit begrenzter Funktion mehr und mehr verändert und sich zu einem umfassenden Steuerungsinstrument der Gesundheitsdienste entwickelt: zu einem bundesgesetzlichen Kollektivsystem mit Rationalisierungs- und Kostendruck, das - zudem immerfort nachbesserungsbedürftig und kaum mehr überschaubar - die ärztliche Berufsfreiheit und individuelle Interessen der Patienten in Bedrängnisse bringt und das Landesrecht verkürzt. Das Buch hat das Verdienst, diesen grundlegend zu überdenkenden Zustand eindrucksvoll vor Augen zu führen, auch wenn die eine oder andere verfassungsrechtliche Deduktion dem kritischen Leser zu wenig feingesponnen sein mag.

\section{Praxisbuch Ethik in der Intensivmedizin.}

Herausgegeben von Fred Salomon. Medizinisch Wissenschaftliche Verlagsgesellschaft, Berlin 2009, $305 \mathrm{~S}$., kart., €44,95

Dem Vorurteil, dass Medizinethik oft durch mangelnden Praxisbezug sehr theorielastig sei, tritt der vorliegende Band resolut entgegen. Der gewählte Titel ,Praxisbuch “ hält, was er verspricht; auf rund 300 Seiten wird das medizinethische Fundament in die klinische Praxis eingebracht. Die lebhafte grafische Aufbereitung empfiehlt eine quasi handbuchartige Benutzung.

Der vorgestellte Sammelband enthält 24 Beiträge von rund $30 \mathrm{Au}-$ toren unterschiedlicher Fachrichtungen und Disziplinen. Vertreten sind neben Experten aus Ethik, Medizin und Pflegewissenschaften auch Juristen, Psychologen und Philosophen; dementsprechend erfreulich perspektiven- und facettenreich ist die Bearbeitung der Bereiche um die angewandte Ethik in der Intensivmedizin. Die Kernbereiche, die in der modernen Intensivmedizin $\mathrm{zu}$ ethischen Handlungskonflikten führen, werden eingängig vorgestellt, und aus der Themenwahl ist bereits der vom Herausgeber intendierte Praxisbezug ersichtlich. Die Auseinandersetzung der Autoren mit ethischen Fragestellungen wirkt besonders authentisch durch die in vielen Beiträgen enthaltenen Fallbeispiele, die auch Leser ohne Praxisbezug zu einer reflexiven Haltung geradezu einladen. Wegen der Beitragsfülle muss die inhaltliche Vorstellung verständlicherweise selektiv ausfallen und sich auf einige besonders markante Kapitel beschränken.

Einen gelungenen Einstieg in die ethische Dimension medizinischen Handelns bietet das erste Kapitel von Maio; hier werden Grundsätze der Medizinethik auf anschauliche Weise erläutert. Der klar strukturierte Überblick und die eingebauten Falldarstellungen führen auch Leser ohne Vorkenntnisse aktueller medizinethischer Debatten in die interessierenden Fragestellungen ein.

Eine umfassende Übersicht wesentlicher Aspekte der rechtlichen Dimension ärztlicher Entscheidungsfindung am Lebensende liefert der Beitrag von Verrel. Zu Recht wird insbesondere auf die terminologisch unbefriedigende Fallgruppenbildung um die Begriffe aktive/ passive und indirekte/direkte Sterbehilfe hingewiesen; die daraus resultierenden Missverständnisse sowie deren Konsequenzen für die medizinische Praxis werden juristisch und ethisch detailliert herausgearbeitet. Angelehnt an die Grundsätze der Bundesärztekammer zur ärztlichen Sterbebegleitung wird eine sinnvolle Alternative zu den aktuell verwendeten mehrdeutigen und interpretationsoffenen Begrifflichkeiten demonstriert. Der Hinweis auf die diesbezüglichen Empfehlungen, die der Stellungnahme des Nationalen Ethikrates von 2006 zu entnehmen sind, wäre zusätzlich dienlich gewesen.

Sold und Schmidt fokussieren auf den Bereich Therapiebegrenzung und -abbruch. Das Kapitel beleuchtet die hauptsächlichen Konfliktsituationen, die sich aus Sicht der klinisch Tätigen in der

Dr. med. Dorothee Dörr, M.A., Fachärztin für Anästhesie, Wiss. Mitarb. am Institut für Geschichte und Ethik der Medizin, Forschungsstelle Ethik, Uniklinik Köln, Deutschland 